\title{
Sensing, Filter and Processing (SFP) Model for Robust Cooperative Position Identification in MANET
}

\author{
Neha Raja Panwar \\ Department of CSE, SDBCT, \\ Indore (M.P.), India
}

\author{
Nitika Vats Doohan \\ Deparment of CSE, SIA, \\ Indore (M.P.), India
}

\author{
Shraddha Kumar \\ Department of CSE, SDBCT, \\ Indore (M.P.), India
}

\begin{abstract}
In MANET is a short range infrastructure independent network with light weight protocols for most of its routing operations. In this radio waves based network the connectivity is taken as key component because in absence of that the transmission is affected. Thus location and mobility information have to be continuously maintained. To identify the position of movable nodes various relative position based approaches is suggested. Identification of accurate location of mobile nodes is a quite heavy task in terms of computation with real time results. Several approach is been suggested to improve the robust position accuracy but well suited in densely connected environment only and can work with a single of dual type of network. This work proposes a novel Sensing, Filter and Processing (SFP) model for robust position identification using different network parameters. At the initial level the approach is proving its efficiency in near optimal calculations and relative position detections.
\end{abstract}

\section{General Terms}

Location Detection, Quadrants System, Coordinates Values, Motion Based Estimation, Detection Accuracy;

\section{Keywords}

Keywords MANET, Location Identification, Cooperative Detection, Tracking, Sensing, Filter and Processing (SFP);.

\section{INTRODUCTION}

Network is having a wide range of devices communicating with each other using various control protocols. By their type of communication and supporting medium they are categorized as wireless and wired network. In wireless medium, mobile ad hoc networks is an emerging technology that has gained a lot of interest due to the wide spreading of mobile devices i.e. personal assistance, net-books, embedded computers, etc. An ad hoc network can be defined as a collection of mobile entities interconnected by a wireless technology, forming a temporary network without the help of any administration or any fixed support [1]. As of now the mobility is desired characteristics for all the users and devices the network is also changing to satisfy such needs. In step to do such position estimation in wireless ad-hoc networks various mechanisms are introduced during the last few decades. The mobile networks are further categorized as infrastructure dependent or independent. Location identification can be a required entity in both the networks and taken as a part of pervasive computing. It is an spontaneous part of wireless network environment which is used in various applications serving our daily needs like office productivity, personal navigations, emergency services and intelligent transportation systems. All it need is the accuracy in estimation with real time updates. Some of the area where the use of such accurate estimation is required is: Vehicular
Position using GPS [2], Flood Alerting and Medicare Services. In both the environment the geographical location needs to be determined for providing help to those affected areas or persons.

Location estimation only works with the specific scheme and type of location information required. Thus the location measurement is of qualitative or quantitative.

Qualitative: In this the Numbers or sets of coordinates are not necessary. Here the Information is processed in the form of location qualifiers or base point with respect to which the position can be given like: in front of the central hall, behind the tower etc. It is sufficient to describe the position of an object or agent. In its application area of robotics, locations described in this form are also known as topological.

$>$ Quantitative: In this type of location detection the coordinates with respect to a map or to an inertial reference system are the result are taken. Here the pure coordinate based description is taken. In this the position descriptions may be absolute, as in the case of latitude and longitude coordinates obtained from a GPS receiver; or relative. In the similar application area of robotics, quantitative location information is also known as metric.

In wireless medium the location identification is known as radio localization or positioning which uses a process for determination of position of target node by using the information of several reference nodes. Mobile ad-hoc networks, cellular networks and wireless sensor networks require localization information.

\section{Location Estimation Categories}

Solutions for localization may be categorized into three groups.

\section{(i) Centralized Localization}

In this type of technique a central authority named as data fusion centre is totally responsible for location identification. It collects all the necessary information for detection of actual position of target node by using a multilateration algorithm. Sometimes, the centralized localization schemes are sometimes unfeasible due to limitations in scalability and reliability.

\section{(ii) Infrastructure-Based Localization}

In this system the previously deployed data fusion center is not required. Apart from that some known position beacons or landmarks are used by Global Positioning System (GPS) which is regarded as infrastructure based localization. Such a classification is possible since satellites send out signals to GPS receivers, which basically compute their location based on the location of the satellites and the distances to them. Cricket is an infrastructure based system in which the beacons send out ultrasound pulses, which may be used by listeners to 
determine their location. Computations are carried out at the client's location, which is the reason why these solutions may be regarded as client-based or mobile-based.

\section{(iii) Cooperative Localization}

It is the most useful and infrastructure less system which required nothing to deployed previously apart from that the Cooperative localization method which is a fully distributed. In this the nodes in a network collaborate together to estimate their position. A collaborative or cooperative localization protocol is based on substitution and averaging of position estimates [3]. Initially, in some cases nodes rely on a few nodes with absolute position information to infer their absolute location. Once the base position of few nodes is identified the relative positions of other nodes can be calculated. It is an incremental approach if the position is determined in a sequence and if it is taken at the same time than the approach is termed as concurrent.

In this approach a novel scheme is proposed to further improvements over the location estimation accuracy. For this various research article had studied and identifies some problems which is solved by the proposed work. Next section give the background and literature study of the paper. After which the problem definition and proposed solution is given. This given solution is measured with some parameters which are also given in the article [4].

\section{BACKGROUND}

In the networked system where the density of the node is very sparse than a centralized solution may not be optimal due to several constraints associated with scalability, robustness and flexibility. It is a well known issues in mobile ad hoc network in which the route information and the configuration is continuously changing over a period of time. Therefore it is required that the computation and identification load is distributed over a networked node for more accurate estimations. This distributed estimation of locations is termed as Cooperative localization. In WSN, cooperative localization works in following environmental conditions: measuring the distance between and known anchor node, identification of positions of each node from an anchor node and refining the positions using the information about the range. The main purpose of sensor localization is to determine the location of sensors in a WSN via noisy measurements. These measurements may include received signal strength (RSS), time-of-arrival, time-difference-of-arrival, and angle-ofarrival. Among the different types of measurements, RSS is a popular method mainly because of its low complexity and cost in software and hardware implementations [4]. It can also be used for cooperative Robot positioning as given in CoCoA [5], which is low-cost, quickly deployable, energy-efficient, and provides reasonable accuracy for the applications.

For measuring the accurate location identification the cooperative localization can be implemented by designing the three main components or functionalities as shown in figure 1. These are Sensing Unit, Processing Unit and Interface. Here sensing is the unit which measure the physical location of the system or landmarked device. It uses further two types of activities: Proprioceptive and Exteroceptive [6]. Proprioceptive devices are used by the system to register its own motion. It uses the sensors which are capable of performing dead-reckoning, which is also known as odometry. It can be assumed that proprioceptive measurements are available at higher sampling rates than measurements from the complementary exteroceptive category. The proprioceptive module is only necessary when nodes possess mobility. Odometry cannot be used for longterm position tracking, due to modeling errors and measurement noise. However, exteroceptive sensors provide for keeping errors, which stem from odometry, within bounds. In navigation terms, sensors of this category are also known as navigation aids.

The second functioning of location identification is the processing unit which allows the combination of data from different sensing unit. This information is processed using different data fusion functions like filters as given in [7]. For accurate positioning of movable nodes with minimum latency occupied by estimation modules requires minimum latency demands and much higher computational powers than a static environment. It can be practically implemented using an learning module which performs the identification of certain parameters such as drift rates of the inertial sensors, particular motion patterns of the host vehicle or node carrier, the nonorthogonality of inertial measurements and lever-arm compensation etc.

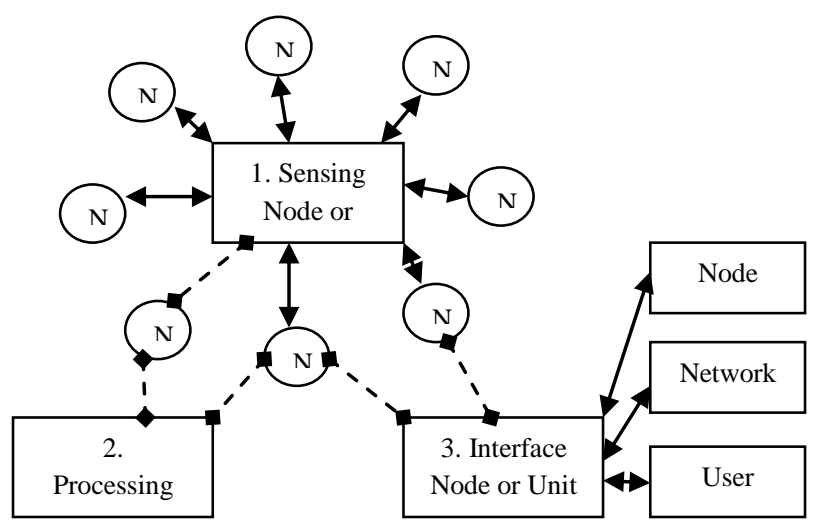

Figure 1: Cooperative Localization Process

Third function is interface for location measurement which is must for users operating or using such location information or updates. This unit passes the measured information to users in a application specific formats. This information may be passed to a user or some other node for further detection of positions and its updates. Thus, the most feasible way to implement cooperative localization is by making each node share its own position estimate. Additional to the position estimate, it is important for other nodes to have a measure of confidence of their position estimate since they may use it in their own position estimation process

\section{LITERATURE SURVEY}

Location identification of mobile node is quite a complicated task during the motion because all the factors of estimation keep changing continuously. Also the transmission is proceeding in according to the estimated positions of source and destinations node. During the last few years several approaches had been suggested to increase the location identification accuracy but some of them is only capable of serving their aim accurately. Few of them have given here.

In the paper [8], a route based robust estimation approach is suggested for cooperative localization using an unknown bounded error model. This framework measures the range and bearing of robots positions for serving as joint solutions. It produces the bounded uncertainty estimates for related configuration of solution. The searching is initiated by measuring the solution satisfying all the localization 
parameters from a configuration space. It can be used for sensors and mobile networks.

Most of the approaches are specific to their operational areas likewise their approach can be specific to that only. Various radio navigation based position estimation is practically applicable of accuracy in location and updates. These operations can be applied by categorizing their areas of estimation like mobile based or network based. To provide the effective identification both the categories had a fixed range of parameters for cooperative localizations. These parameters are Angle Of Arrival (AOA), Time Of Arrival (TOA), Time Difference Of Arrival (TDOA), Received Signal Strength (RSS), Cell ID based positioning, Fingerprinting etc [9]. Here some of the cooperative positioning mechanism is also proposed like Data-fusion and cooperative filtering.

The paper [10], suggest some unique mechanism based on pairwise cooperative measurement which combining estimate the velocity and position of the mobile node. It includes various parameters to identify the p[position more accurately and for this the approach uses radial velocity calculation and state space based distributed Kalman filter model. The approach is occupying limited resources in terms of computation and bandwidth. Primarily it designs a Gaussian estimation bound for developing a centralized and decentralized Kalman filter. Numerical analysis of the suggested approach using Cramar Rao function proves its effectiveness. Initial measurement of the accuracy for the approach will shows the approach and its outlined benefits for cooperative localization.

For further accuracy and reducing the computation overhead different authors designs multiple functions and filters for information process and location identification. Likewise a model proposed using Bayesian Network in [11] with proximity measurement. It uses the deployment knowledge and information for description of sensor nodes using probabilistic graphical model in grid based topology. Based on the above location model the CLPKBN scheme for cooperative localization is proposed. Result evaluation of the above approach is proven by Probability Grid scheme on simulator and measures the effective results.

Some of the approaches also use the mined network information from which the estimation has to be applied. Taking the above phenomenon, the paper [12] suggested a apriori based position estimation which is more accurate. It takes the base node whose position is fixed and the moving nodes position is identified in accordance to that fixed node. It exploited to perform Bayesian estimation procedure. Experimental evaluation of the proposed technique outperforms the maximum existing approaches. Instead of above function the approach uses a mathematical model based on Markovian property for correct estimation.

The paper [13] gives application specific approach for multirobot localization problem. It uses the unknown bounded by which it able to define convex polytopes in the configuration space. Estimates for the uncertainty in a range of parameters of the team's pattern such as the fixed position of a single robot, or the relative positions of two or more nodes can be obtained by projecting this polytope onto suitably chosen subspaces of the configuration space. The paper proposes a novel approach to approximating these projections using linear programming techniques. The approach can handle both bearing and range measurements with a computational complexity scaling polynomials in the number of robots. Finally, the workload is readily distributed - requiring only the communication of sensor measurements between robots. Here the simulation verifies results for this approach implemented on a multi-robot team.

To design an accurate and precise location measurement framework some design rules needs to be taken as fixed and other are fluctuating. The environment for which the location detection is required needs to provide the ubiquitous information about the factors and parameters affecting the positions frequently. In the work presented in [14] authors conclude why several existing localization systems are unsatisfactory for this application domain and suggested a new system called Calamari. Preliminary experiments show that this ranging error with this algorithm is sufficient to give rise to position estimates that are on average $20 \%$ of the maximum distance between connected nodes in the network.

Among many localization methods, the hop-count based approach given in [15] is simple and scalable. The above paper suggest a auction based task allocation scheme by which the robots can negotiate with the static sensor nodes and then select the most suitable robots to move to the area of sparse node density from which the location accuracy can be increased. It can be taken for both static and dynamic nodes as a base of cooperative position identification. The approach suggest a guideline of better accuracy as the motion of robot must be form sparse density region from which the precision can be improved.

Some of the papers had focused their concerns towards the distance measure. According to which if the signal strength is weak than the distance is high. Thus the work in [16] addresses the problem of position localization of mobile nodes in ad hoc wireless networks based on received signal strength indicator (RSSI) measurements. Here the node mobility is designed as a non-linear system driven a Coordinated Turn Model (CTM). The approach also uses Kalman filter for self localization and estimation refinement. Here the variants of Kalman filter are used for higher accuracy. AT the performance based evaluation the above variants is serving as a great option in near future.

In the paper [17] a novel distributed cooperative localization algorithm for indoor WSNs is given. Here a mechanism Cooperative Localization with Quality of estimation (CLOQ) integrates and disseminates the quality of the TOA ranging and position information. It has the ability to reduce the effects of the cluttered indoor environments by measuring and justifying the associated ranging errors. Apart from that the information regarding the integrity of the position estimate is further incorporated in the iterative distributed localization process which further reduces error escalation in the network. The simulation results of CLOQ algorithm are then compared against the derived G-CRLB, which shows substantial improvements in the localization performance.

Localization is one of the most crucial capabilities of autonomous robots. Cooperative localization has been proved to be successful in multi-robot localization. Yet, nearby moving objects could humiliate the cooperative localization performance. In the paper [18], authors demonstrate that the cooperative simultaneous localization and tracking approach is superior in challenging scenarios. Localization and moving object tracking are mutually beneficial. The proposed approach is evaluated using humanoid robots in the RoboCup environment in which only uncertain data from onboard cameras and odometry are used. Experimental evaluation of results proves the accuracy and flexibility of vision-based cooperative simultaneous localization and tracking algorithm. 


\section{PROBLEM DEFINATION}

After studying the various approaches used for location identification it is found that the detection must be operated by some intermediate node in MANET and by some reference points in WSN. This detection involves the sensing of the basic information or base point positions which later on processed by processing elements. This processing involves the different mechanism for transformation of data. Designing of this module will serve the functionality of data categorization, extracting the useful information, applying the filer mechanism getting the directional intimations and updating the devices. After this modification or updations of recent generated directional intimations the information is transferred to the user, device or some other node. The above steps is used for localization improvements even though there are some issues which remains unaddressed or solving some problem will raises some other associated difficulties. It needs to be solved effectively with lesser computational load, time and complexities. Also the presence of noise in the localization is comes under NP-Hard problem domain. The problem I which the work focuses its intensions are:

Problem 1: Identification of correct amount of location information at local level and in distributed level with frequent exchanging mechanism.

Problem 2: Effective mechanism is required to deal with changing coverage criteria's and variable base points.

Problem 3: The cooperative localization will not be effectively updated by sudden change in velocity of nodes and topology of the network. Thus it is unable to measure these abrupt changes.

Problem 4: Refining of states is not available with any of the existing mechanism as a integrated mechanism.

Problem 5: Classification of non-stationary signal jamming is not properly defined. It raises a problem when the received signal strength is jammed due to certain reasons than the estimation is wisely affected by it.

Hence above mentioned issues are the some basic considerations for this work to be drafted or proceeds in a specific directions. It also focuses on the frequent updations of the information and accuracy in location estimations. Also the work tries to embed such developed codes in existing simulators in near future.

\section{PROPOSED SFP SOLUTION}

For solving the above mentioned issues multiple methods are overlooked as an aspect of localizations solution. It also helps in understanding the network parameters affects the resulting position of error behaviour and scalable information. This work takes the multiple information as a input like network geometry and topology, density of node, ranging accuracy information, anchor or base node uncertainty etc are the factors affecting the quality of estimations. Here the location information is essential for increasing the efficiency in further estimation of nodes position accurately. It can be widely used for implementing the different network management, addressing, power aware and location based routing decisions. Thus this work proposes a novel sense, filter and processing (SFP) operation based estimation model for improved robust position identification for cooperative localization.

The suggested approach has three basic modules for cooperative localization:

Sense Module: In this phase the sink node collects the information from various nodes or sensor units. Initially, the units transmit their coordinate's information in static condition. This information is broadcasted to every neighbour for relative position measurement.

Filter Module: This phase gathers all the information received from various nodes and sensors. It collects some other relative parametric information like: received signal strength (RSS) of each receiving signal of nodes, time of flight $(\mathrm{ToF})$ which is measured by sink node, probe request and reply TTL fields and formation of fix shape detections. All the above values is required or accuracy in location identification relative to some other node whose location information is already available

Processing Module: It is the position measurement modules used to apply certain decisions about the relative directional movement of the nodes. From the above information this modules process the data for identifying the relative motion of node in specific direction. From this calculation the new coordinates is measured and whose updated values is given by massage. When a node receives this message about the position change in other nodes motion than it updates the respective stored information.

In the above manner the whole process continues the updations criteria's in network topologies.

\section{Working Scenario:}

As the suggested approach is used for cooperative localization and hence the modification is the positions of the node must be frequent and acquire less computational loads. 


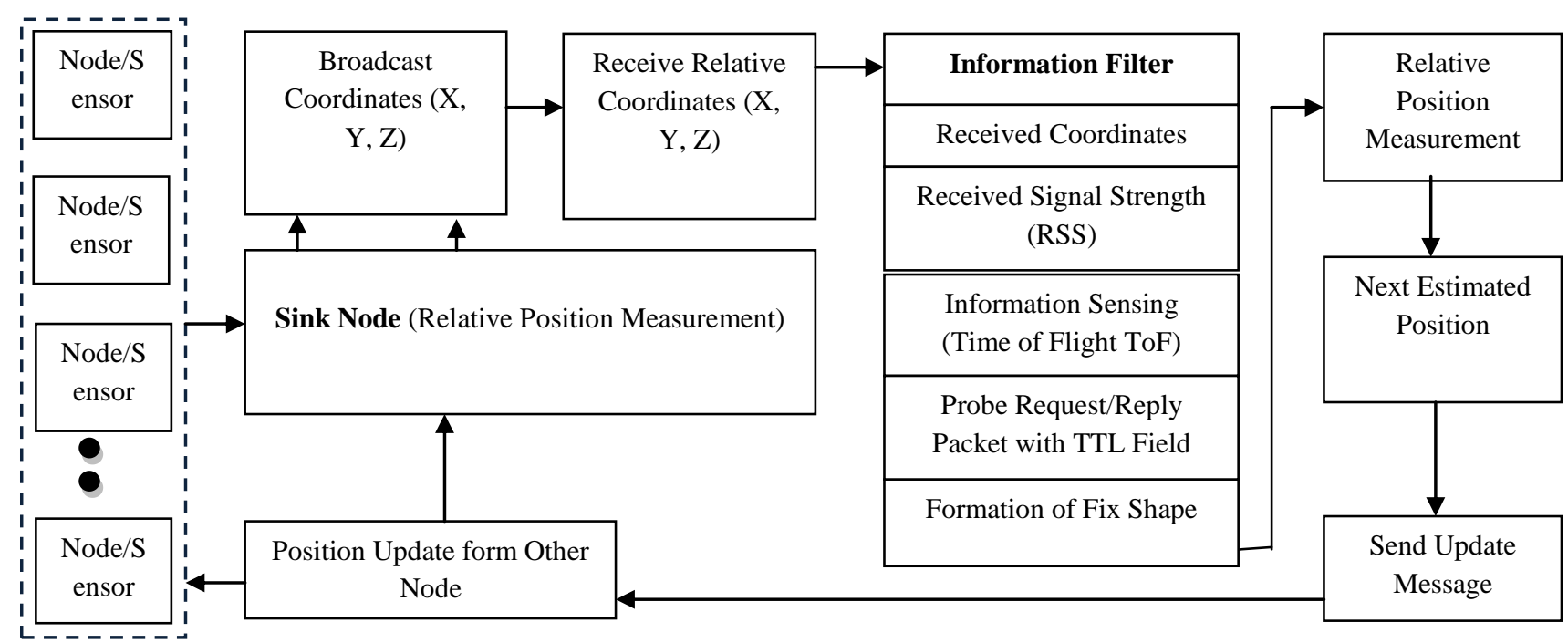

Figure 2: Sense, Filter and Process (SFP) Operation Based Robust Location Estimation Model

If the load of positions detection messages is very high then the actual transmission and mobility of nodes might be affected. For implementing the above solution first the nodes with specified network range has to be created using any network simulation environment. The given solution will give effective results and the accuracy is also like to be improved in near future so the proximity value of this work is taken as a range of $5 \%$.

\section{Coordinates Receive $(x, y, z)$}

Receive signal strength (RSS) (70-100\% Distance $=0-0.5 \mathrm{~m}$, $50-70 \%$ Distance 0.5-1 m, 40-50\% Distance 1-1.5 m, 3040\% Distance 1.5-2 m, 20-30 \% Distance 2-2.5 m, 10-20\% Distance 2.5-3.5, 5-10\% Distance 3.5- $6 \mathrm{~m}, 0-5 \%$ Distance 6-8m)

The above values are of the form coordinates of three dimensional axes. Later one is used to represent the distance of node form sink or relative node taken as strength variable.

Time of Flight (ToF) is the parameter used to describe the inertial measurement in the position of node. Initially the value of ToF is zero but as the node start moving the time based motion is considered by the sink node.

Probe Request/ Probe Reply packets are used to detect the TTL values between the Hops available within the range of the network where the location identification is working.

Formation of Fixed Shape is unique relative motion in which all the nodes move simultaneously with respect to each other in a specific formation previously available or designed. These formations will also help the relative position detection each and more frequent.

Thus by the above methods implementation the approach is proving its practical feasibility in near future. Thus the operational results will also verify that the efficiency of the suggested approach will going to be great after actual implementation. There are so many other parameters such as angle of arrival (AoA), Time of arrival (ToA), time difference of arrival (TDoA) which will be taken in near future for practical applicability of the approach.

\section{Application Area}

(i) The approach also had wide applicability area such as:

(ii) Flood Recovery and Help Management

(iii) Disaster Recovery and Help Management

(iv) Emergency Health care Required

(v) Transportation system using GPS

(vi) Navigational System

(vii) Military Tracking applications etc.

\section{Performance Evaluation Factors}

RMSE (Root Mean Square Error): This parameter will serve to detect the performance of location estimation in terms of accuracy near by the actual position and identified position. If the difference is less means the approach will serve its aim.

Computational Overhead: To identify the location updates frequently the number of steps in processing will increases the burden of computation on each node which reduces the battery life. This will be again a performance parameter.

Time of Detection: This is the time taken to detect the position changes in the nodes locations. If the time of information is near to real time than the approach will proves its effectiveness.

Localization Error: It is the cumulative values which depend upon the above identified parameters. Localization error must be reduced with number of phases of approach and increased nodes and sensor in the network.

\section{EXPECTED OUTCOMES}

There are number of reasons for using location identifications with more accuracy. In terms of the suggested approach following are the some expected outcomes after implementation:

$>$ Reducing the noise in previously identified location information.

$>$ Sensed data is useless when there is no field acquiring the data. Same is the case with broadcasted messages if the location updations is transferred with route discovery 
than the broadcasting will be only in restricted direction thus saves the unnecessary process.

> Target tracing application can widely uses such system because the accuracy of location or position detection is the prime aim same is with the suggested approach.

$>$ Designing the filter will increase a computational load on the nodes. Thus in this work no extra functionality is assigned to the separate nodes instead of that same information is processed in such a way to improve location detections.

$>$ Energy saving operations required the accurate position estimates so as serving by the suggested mechanism.

$>$ Limited bandwidth and memory requirement and works well in densely connected topology situation.

$>$ Decentralized approach will reduces the operational load.

\section{CONCLUSION}

In this paper a novel approach SFP is been proposed for improving the location accuracy in ad-hoc wireless network. It is a combined model which works well in both the cases of MANET and WSN. Along with its applicability the initially it is identified that the approach will consume lesser resources than the other existing mechanism. The approach is based on three phases sensing, filtering and processing after which the location updates can be generated which is more accurate in terms of RMSE values. Detection is also serving the principle needs of optimality. It is capable of detecting the correct position in both static and dynamic environment. The paper also discusses the outlier analysis with some error bounded values as a robust estimation process. Final comment on the approach will be given here by the author as:

"Effective, Efficient and Lightweight Robust Position Estimation Serving the needs in terms of less energy requirement and computation loads"

\section{REFERENCES}

[1] Zafoune Youcef, Mokhtari Aicha and kanawati Rushed, 2010.Mobile Code localization in ad hoc network: A comparative study of centralized and distributed approach. In IJCNC, Vol 2, No. 2, March 2010.

[2] Boukerche Azzedine, Oliveira, Horacio A.B.F., Nakamura, Eduardo F., Loureiro, Antonio A.F. 2008. Vehicular Ad Hoc Networks: A New Challenge for Localization-Based Systems. In Elsevier Journal of Computer Communication. doi:10.1016/j.comcom.2007.12.004.

[3] Vukadinovic Vladimir and Mangold Stefan, 2012. Performance of Collaborative GPS Localization in Pedestrian Ad Hoc Networks. In ACM MobiOpp 12, doi: 978-1-4503-1208-0/12/03, March 2012.

[4] Michael Buehrer, R. 2014. Cooperative and noncooperative localization. In non-line-of-sight environments, research Articl.,

[5] Dimitrios Koutsonikolas Saumitra M. Das Y. Charlie Hu Yung-Hsiang Lu C.S. George, Lee, CoCoA: Coordinated Cooperative Localization for Mobile Multi-Robot Ad Hoc Networks. In School of Electrical and Computer Engineering, Purdue University, USAby National Science Foundation under Grant IIS-0329061.
[6] Fontanella Diana, Bauernfeind Roland, Essfellar Bernd and Dr. Henn Gunten, 2013. In Car GMSS Jammer localization using Vehicular Ad-Hoc Network, in Inside GNSS Research Society, June 2013.

[7] Lhomme Edouard, Frattasi Simone and Schwefel Hans Peter. Enhancement of Localization Accuracy in Cellular Networks via Cooperative AdHoc Links. In Center for TeleInFrastruktur (CTIF), Aalborg University, Denmark.

[8] Taylor Camillo J. and Spletzer John. A Bounded Uncertainty Approach to Cooperative Localization Using Relative Bearing Constraints. In Department of Computer and Information Science, University of Pennsylvania, USA.

[9] Rosa1 Francescantonio Della, Leppäkoski1 Helena, Ghalib Ata-ul. Ad Hoc Networks for Cooperative Mobile Positioning. At IntechOpen Online Research Community of Mobile Ad-Hoc Networks: Applications.

[10] Dong Liang, 2012. Cooperative Localization and Tracking of Mobile Ad-Hoc Network, in IEEE Transaction of Signal Processing. ISSN: 1053-587X, Vol 60, No 7, July 2012.

[11] Lo Shih-Hsiang, Wu Chun-Hsien and Chung Yeh-Ching. Cooperative Localization with Pre-Knowledge Using Bayesian Network for Wireless Sensor Networks. Partially supported by NSC 95-2221-E-007-018 and MOEA 95-EC-17-A-04-S1-044. Dept of Computer Science NTHU.

[12] Caleffi Marcello, Ferraiuolo Giancarlo, Paura Luigi. A Bayesian Location Estimation Technique for Mobile Ad Hoc Networks. In National project Wireless 8O2.16 Multi-antenna mEsh Networks (WOMEN) under grant number 2005093248.

[13] Spletzer John R. and Taylor Camillo J. A Bounded Uncertainty Approach to Multi-Robot Localization. In GRASP Laboratory - University of Pennsylvania, Philadelphia, PA, 19104, USA.

[14] Dean Cameron, 2012. The Design of Calamari: an Adhoc Localization System for Sensor Networks. In University of California at Berkeley.

[15] SIT Terence Chung Hsin, LIU Zheng, ANG Jr, Marcelo $\mathrm{H}$. and SEAH Winston Khoon Guan. Multi-Robot Mobility Enhanced Hop-Count Based Localization in Ad-Hoc Networks. In National University of Singapore.

[16] Murillo Julian Alberto Patino, 2011. Kalman-based schemes for mobile nodes localization in ad-hoc networks. In University of Nacional, Colombia.

[17] Alsindi Nayef , 2008. Indoor Cooperative Localization for Ultra Wideband Wireless Sensor Networks. In PHD Thesis at Worcester Poly-technique Institute.

[18] Chang Chun-Hua, Wang Shao-Chen and Wang ChiehChih. Vision-Based Cooperative Simultaneous Localization and Tracking. In Graduate Institute of Networking and Multimedia, National Taiwan University, Taiwan. 